\title{
Capability of Radial- and Convex-Arrayed Echoendoscopes for Visualization of the Pancreatobiliary Junction
}

\author{
Yoshihide Kanno, Kei Ito, Shinsuke Koshita, Takahisa Ogawa, Hiroaki Kusunose, Kaori Masu, Toshitaka Sakai, Toji Murabayashi, \\ Sho Hasegawa, Fumisato Kozakai, Yujiro Kawakami, Yuki Fujii and Yutaka Noda
}

Sendai City Medical Center, Sendai, Japan

Background/Aims: Although both radial- and convex-arrayed endoscopic ultrasonography (EUS) scopes are widely used for observational EUS examinations, there have been few comparative studies on their power of visualization. The aim of this study was to evaluate the capability of these EUS scopes for observation of the pancreatobiliary junction.

Methods: The rate of successful visualization of the pancreatobiliary junction was retrospectively compared between a radial-arrayed and a convex-arrayed echoendoscope, from a prospectively maintained database. Study periods were defined as January 2010 to December 2012 for the radial group, and February 2015 to October 2016 for the convex group because the respective scope was mainly used during those periods.

Results: During the study period, 1,660 cases with radial EUS and 1,984 cases with convex EUS were recruited. The success rates of observation of the pancreatobiliary junction were $80.0 \%$ and $89.5 \%$, respectively $(p<0.0001)$.

Conclusions: The capability of visualization of the pancreatobiliary junction in observational EUS was found to be better with a convex-arrayed than with a radial-arrayed echoendoscope. Clin Endosc 2018;51:274-278

Key Words: Endosonography; Radial; Curved linear; Pancreatobiliary maljunction

\section{INTRODUCTION}

Since its development in the 1980s, the role of endoscopic ultrasonography (EUS) in pancreatobiliary diseases has rapidly expanded. EUS can detect small biliary stones, ${ }^{1-4}$ reveal pancreatobiliary maljunction, ${ }^{5,6}$ and can be used for the follow-up of some minor anomalies without any invasive or less readily available examinations. The international consensus guidelines for the management of intraductal papillary mucinous neoplasms (IPMNs) ${ }^{7}$ recommend that EUS should be performed for checking indications for surgery, when there

Received: June 14, 2017 Revised: July 11, 2017

Accepted: August 20, 2017

Correspondence: Yoshihide Kanno

Sendai City Medical Center, 5-22-1 Miyagino, Sendai 983-0824, Japan Tel: +81-22-252-1111, Fax: +81-22-252-9431, E-mail: yoshi-hk@openhp.or.jp ORCID: https://orcid.org/0000-0002-2044-5483

(c) This is an Open Access article distributed under the terms of the Creative Commons Attribution Non-Commercial License (http://creativecommons.org/ licenses/by-nc/3.0) which permits unrestricted non-commercial use, distribution, and reproduction in any medium, provided the original work is properly cited. are worrisome features, and for follow-up when IPMNs are greater than $2 \mathrm{~cm}$ in size. Moreover, it has been used for many interventions, such as tissue sampling, drug injection, drainage, and creation of an anastomosis via the intestinal wall.

For observation, including diagnosis and follow-up, two types of echoendoscope (ES) are commonly used: radial-arrayed and convex-arrayed. They differ in the plane of image obtained to the axis of the endoscope (perpendicular or parallel) and in the viewing angle (360 or 180 degrees). A radial-arrayed ES (radial ES) has a 360-degree viewing angle, enabling easy identification of surrounding organs and vessels, compared to a convex-arrayed ES (convex ES). ${ }^{8}$ Due to the difference in viewing plane, radial ES is able to provide a longitudinal image of the pancreas, gallbladder, and extrahepatic bile duct, whereas convex ES is thought to provide better visualization of the perihilar bile duct, neck of the pancreas, and the vascular system in the upper abdominal cavity. ${ }^{8,9}$

However, few comparative studies about the degree of visualization between these types of EUS have been performed so far. We thus carried out a comparative study using a pro- 
spectively maintained database at our center to evaluate the visualization capability of these two scopes.

\section{MATERIALS AND METHODS}

\section{Patients}

At Sendai City Medical Center, almost all observational EUS had been performed with radial ES until 2012. After a transitional period of few years, the use of convex ES was instituted and has continued until date. The ES mainly used was GF-UM2000 (Olympus Co., Tokyo, Japan) before 2012 and GF-UCT260 (Olympus Co.) after 2015. In almost all procedures, patients desired and received sedation with diazepam or midazolam, combined with pentazocine if necessary.

Consecutive patients who underwent observational EUS for the pancreas or the biliary system with a radial EUS between January 2010 and December 2012 (radial group) and those who underwent EUS with a convex ES between February 2015 and October 2016 (convex group) were included, and retrospectively compared in this study. The ultrasound processor utilized was EU-ME1 in the radial group and EU-ME2 in the convex group. The following cases were excluded from this study: (1) cases with surgically reconstructed anatomy of the upper digestive system (cases with Billroth-I reconstruction were not excluded), and (2) interventional EUS cases.

\section{Outcome measurements}

All EUS procedures were performed by experts with an experience of more than 1,000 EUS examinations, or trainees under the strict supervision of experts. The EUS experience of experts ranged from 5 years to more than 30 years. Findings and results of the examinations were judged by an expert or by a trainee under the strict supervision of an expert.

At our center, it is mandatory that endoscopists must attempt to observe and estimate the form of the pancreatobiliary junction (PB junction) in all observational EUS cases. In cases where visualization of the PB junction is not essential, such as pancreatobiliary maljunction or ampullary neoplasm, visualization attempts can be omitted if prolongation of the examination seems unreasonable due to pain or the need for additional sedatives. The prospectively maintained database for EUS at our center includes the degree of visibility of the PB junction: well observed, observed but not clear, or not observed (Fig. 1).

"Well observed" was defined as a result, in which the distal ends of both, bile duct and main pancreatic duct were clearly observed, with visibility of the positional relation between these ducts, the duodenal ampulla, and the duodenal muscular layer. It does not necessitate visualizing both the ducts in one image at the same time.

Cases in which both these ducts were barely visible, but the definite relation between them or that between ducts and the duodenal structures (the ampulla and muscular layer) was not clearly recognized, were defined as "observed but not clear". These cases included: those in which the true ends of both ducts could not be clearly recognized with the pushed position of the scope from the duodenal bulb; those in which
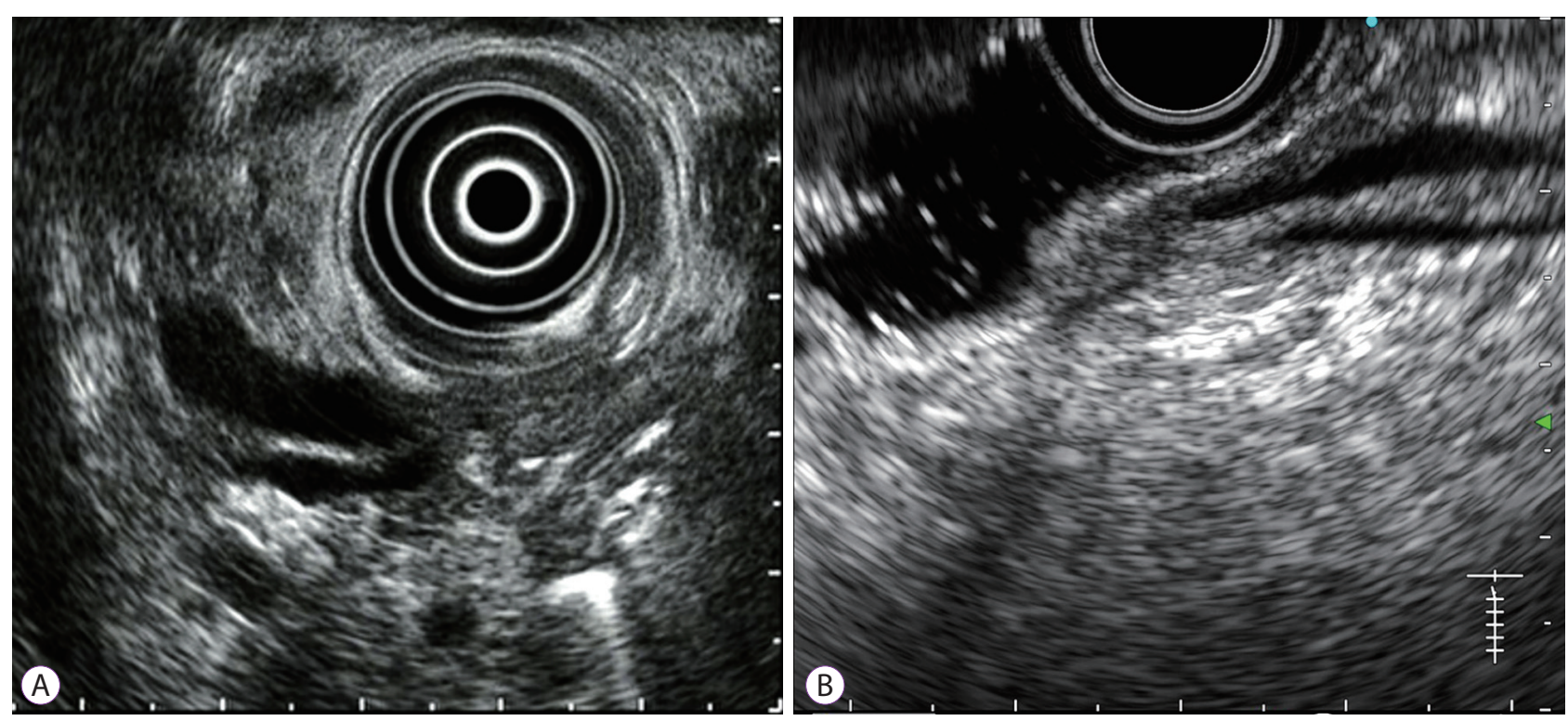

Fig. 1. "Well observed" endoscopic ultrasonography (EUS) views of the pancreatobiliary junction. "Well observed" was defined as a case in which the distal ends of both the bile duct and the main pancreatic duct were clearly observed with awareness of positional relation between these ducts, the duodenal ampulla, and the duodenal muscular layer. It does not require visualization of both the ducts in one image at the same time, especially with convex EUS. (A) View by a radial-arrayed echoendoscope. (B) View by a convex-arrayed echoendoscope. 
the structure of the ampulla was barely recognizable as the ampulla was deformed by pressure of the scope; and those in which the ends of the ducts were collapsed due to such pressure, although the bile duct and the pancreatic duct were barely visible.

When the distal end of either the bile duct or the pancreatic duct could not be identified, the case was considered "not observed".

Success in visualization of the PB junction was defined when evaluated as "well observed" in this study. The main outcome measurement was designated as the success rate of visualization of the PB junction with the ES which was mainly used during each period, i.e., GF-UM2000 in the radial group and GF-UCT260 in the convex group.

\section{Analytic methods}

After identification of all EUS cases, cases which met the exclusion criteria, those in which observation of the PB junction was not attempted, and those in which the result of visualization of the PB junction was not available were eliminated. The remaining cases were retrospectively compared between the radial and convex groups.

Fisher's exact test, Welch's $t$-test, and the Chi square test were applied for analyses.

An appropriate sample size was calculated to be 945 for each group to provide a power of $80 \%$, to detect a difference between $80 \%$ and $85 \%$ with a two-sided significance of 0.05 .

A $p$-value of less than 0.05 was considered to be significant.

SPSS software version 24 (IBM Japan Ltd., Tokyo, Japan) was used for all analyses.

\section{Ethics}

Informed consent for EUS examination was obtained from all patients before the procedure.

This study was approved by Sendai City Medical Center Institutional Review Board.

The registration ID issued by UMIN was UMIN 000025254.

All authors had access to the study data and reviewed and approved the final manuscript.

\section{RESULTS}

The initial number of identified patients who underwent observational EUS during the study period of the radial group with GF-UM2000 and of the convex group with GFUCT260 was 1,782 and 2,190, respectively. After elimination of cases in which visualization of the PB junction was not attempted for some reason, such as patient's non-cooperation due to inadequate sedation, and those in which the prospective data was unavailable, 1,660 patients in the radial group and 1,984 patients in the convex group were finally analyzed. Patients' characteristics are shown in Table 1. There was no difference in any of the baseline parameters between the 2 groups. There were 46 patients (2.7\%) with Billroth-I reconstruction in the radial group and $60(3.0 \%)$ in the convex group.

The number of endosonographers was 8 experts and 6 trainees in the radial group, and 5 experts and 6 trainees in the convex group. In the radial group, 721 cases (43\%) were completely performed by experts, 402 (24\%) were performed by experts after initial observation by trainees, and 537 (32\%) were performed by trainees under the strict supervision of experts. In the convex group, these numbers were 799 (40\%), 450 (23\%), and 735 (37\%), respectively.

The success rate of visualization of the PB junction was

Table 1. Patients' Characteristics

\begin{tabular}{|c|c|c|c|}
\hline & $\begin{array}{l}\text { Radial group } \\
\quad(n=1,660)\end{array}$ & $\begin{array}{l}\text { Convex group } \\
\quad(n=1,984)\end{array}$ & $p$-value \\
\hline Sex, male & $945(60.0 \%)$ & $1,180(59.5 \%)$ & 0.12 \\
\hline Age, years +/- SD & $67.0+/-12.3$ & $66.5+/-11.5$ & 0.20 \\
\hline Main indication for EUS & & & n.s. \\
\hline IPMN, pancreas cysts & $791(4737 \%)$ & 987 (49.7\%) & \\
\hline Gallbladder abnormalities & $341(20.5 \%)$ & $371(18.7 \%)$ & \\
\hline Bile duct abnormalities & $179(10.8 \%)$ & $245(12.3 \%)$ & \\
\hline Screening & $98(5.9 \%)$ & $128(6.5 \%)$ & \\
\hline Pancreas abnormalities except IPMN & $111(6.7 \%)$ & $115(5.8 \%)$ & \\
\hline PB junction or ampullary abnormalities & $28(1.7 \%)$ & $20(1.0 \%)$ & \\
\hline Other & $112(6.7 \%)$ & $118(5.9 \%)$ & \\
\hline
\end{tabular}

SD, standard deviation; EUS, endoscopic ultrasonography; IPMN, intraductal papillary mucinous neoplasm; PB junction, pancreatobiliary junction; n.s., not significant. 
Table 2. Results of Visualization of the Pancreatobiliary Junction

\begin{tabular}{lccc}
\hline & $\begin{array}{c}\text { Radial group } \\
(\boldsymbol{n}=\mathbf{1 , 6 6 0 )}\end{array}$ & $\begin{array}{c}\text { Convex group } \\
(\boldsymbol{n}=\mathbf{1 , 9 8 4 )}\end{array}$ & $\boldsymbol{p}$-value \\
\hline Well-observed & $1,328(80.0 \%)$ & $1,776(89.5 \%)$ & $<0.0001$ (vs. all others) \\
Observed but not clear & $193(11.6 \%)$ & $159(8.0 \%)$ & \\
Not observed & $139(8.4 \%)$ & $49(2.5 \%)$ & \\
\hline
\end{tabular}

$80.0 \%$ in the radial group and $89.5 \%$ in the convex group, with a statistically significant difference $(p<0.0001)$ (Table 2$)$. The number of cases in which pancreatobiliary maljunction was detected in previous examinations or in the EUS study was 11 in the radial group and 7 in the convex group, and the junction was successfully visualized in all such cases.

\section{DISCUSSION}

Radial-arrayed and convex-arrayed ESs are substantially different in degree of visualization of any particular view, because of the difference in viewing plane. It is relatively easy to visualize a wide area of the pancreas body and tail in one view with a radial ES, with a longitudinal view of the splenic vein or artery, which helps in recognizing a certain orientation. Radial ES is also advantageous in obtaining a view with a greater length of the extrahepatic bile duct. Such organs are visualized in a short axis with convex ESs, resulting in difficulty in identifying the location on a still image, in the absence of a moving image. On the other hand, it is often more difficult to comprehend an anatomical orientation in the upper portion of pancreas head to neck, with radial ES than with convex ES. Anatomical orientations are relatively understood easily in any area, with convex ES, by real-time estimation with scope manipulation.

Although the two types of scopes differ in some aspects, there have been few comparative studies between them. The reason might have been the difficulty in designing studies to evaluate differences, since both types of EUS can be used for examinations in almost all cases by expert endosonographers. Randomized studies would require a large sample size, involving a lot of work and a long study period. Moreover, in daily practice it would be extremely difficult to have both types of ES available at the start of examinations in all qualified patients, unless the study institution had 2 or more expensive scopes for each type.

The sole and well-designed randomized controlled trial by Kaneko et al. ${ }^{10}$ evaluated the degree of visualization of 11 defined points in the pancreatobiliary region, including several portions of the pancreas, the bile duct, some vessels, and the major papilla. They compared radial ES and convex ES in 200 patients, and demonstrated superiority of the former in terms of overall capability. Regarding visualization of the papilla, the rate of highest score on a scale of 3 grades, was also higher in the radial ES group ( $41.4 \%$ vs. $18.8 \%, p=0.001)$. Although the reasons for the difference between Kaneko's study and our study are unclear, different definitions of the outcomes might be related to the difference. The evaluated outcome in their study, which was most similar to that of our study, was successful visualization of the "papilla" with "images" of the pancreatic and bile ducts penetrating the duodenal muscularis propria. In our study, however, successful visualization of the "PB junction" was based on "recognition" of the distal ends of both ducts rather than on images of the ducts. Moreover, although visualization of both the ducts in one image at the same time was not required in our study, Kaneko's study did not address this issue. Since they defined each score by the obtained "image", successful cases with the highest score might not have included those in whom still images with both the ducts could not be obtained.

In the present study, convex ES were found to be easier for observation of the PB junction than radial ES. However, it is possible that the motivation to locate the $\mathrm{PB}$ junction could have differed between the analyzed study periods. Although it is difficult to verify the equivalence and the comparability, we have had a policy of observing the PB junction in all patients since the earliest days of endosonography; therefore, the difference in motivation is unlikely. In addition, all endoscopists in the study period of the radial group had been long accustomed to and familiar with radial ES. Before the study period, some experts had seen the emergence of ES, and strove to find better techniques of observation, and other experts learned such techniques with radial ES as trainees under the guidance of senior experts. Techniques of convex ES were learned after the experts had completely acquainted themselves with radial ES. This background would have been advantageous for radial ES, and the result of the study, which indicated the superiority of convex ES seems natural.

However, the higher capability of visualization of the PB junction does not necessarily indicate that convex ES are always better than radial ES. For visualization of other sites, the superiority between the two systems remains unknown. Moreover, the higher capability of visualization should not 
be equated with the capability of evaluation of diseases. It is also unknown as to which EUS is better at estimation of the extent of malignant tumors, the diagnosis of irregularity of a ductal wall, the form of pancreatobiliary maljunction, and the presence of choledochocele. However, the extent of an ampullary cancer can be well evaluated with a radial ES. ${ }^{11}$

This study had some limitations. It was a retrospective study in a single center. Moreover, the study periods were completely different between the two compared groups. The medical personnel involved, including not only endoscopists but also nurses, assistants, and trainees, were partly different. Equipment other than scopes, such as a display monitor and a universal ultrasound processor, were somewhat different between the groups. These limitations could have influenced the results in some cases, but the outcome of this study is still informative due to the large study population, exceeding the calculated sample size required for detection of a difference and significance with an extremely small $p$-value.

In conclusion, the capability of visualization of the $\mathrm{PB}$ junction in a setting of observational EUS was found to be better with a convex-arrayed EUS compared to radial-arrayed. Although the generalization of this result to organs other than the PB junction is unknown, convex-arrayed EUS are possibly easy to handle and understand, resulting in earlier mastery by trainees. Learning to use convex scopes should perhaps be given priority because they can be applied for interventional EUS, unlike radial ones.

\section{Conflicts of Interest}

The authors have no financial conflicts of interest.

\section{REFERENCES}

1. Sugiyama M, Atomi Y. Endoscopic ultrasonography for diagnosing choledocholithiasis: a prospective comparative study with ultrasonography and computed tomography. Gastrointest Endosc 1997;45:143-146.

2. Kondo S, Isayama $\mathrm{H}$, Akahane $\mathrm{M}$, et al. Detection of common bile duct stones: comparison between endoscopic ultrasonography, magnetic resonance cholangiography, and helical-computed-tomographic cholangiography. Eur J Radiol 2005;54:271-275.

3. Mirbagheri SA, Mohamadnejad M, Nasiri J, Vahid AA, Ghadimi R, Malekzadeh R. Prospective evaluation of endoscopic ultrasonography in the diagnosis of biliary microlithiasis in patients with normal transabdominal ultrasonography. J Gastrointest Surg 2005;9:961-964.

4. Ney MV, Maluf-Filho F, Sakai P, Zilberstein B, Gama-Rodrigues J, Rosa $\mathrm{H}$. Echo-endoscopy versus endoscopic retrograde cholangiography for the diagnosis of choledocholithiasis: the influence of the size of the stone and diameter of the common bile duct. Arq Gastroenterol 2005;42:239243.

5. Mitake M, Nakazawa S, Naitoh Y, et al. Value of endoscopic ultrasonography in the detection of anomalous connections of the pancreatobiliary duct. Endoscopy 1991;23:117-120.

6. Sugiyama M, Atomi Y. Endoscopic ultrasonography for diagnosing anomalous pancreaticobiliary junction. Gastrointest Endosc 1997;45:261-267.

7. Tanaka M, Fernández-del Castillo C, Adsay V, et al. International consensus guidelines 2012 for the management of IPMN and MCN of the pancreas. Pancreatology 2012;12:183-197.

8. Katanuma A, Maguchi H, Osanai M, Takahashi K. The difference in the capability of delineation between convex and radial arrayed echoendoscope for pancreas and biliary tract; case reports from the standpoint of both convex and radial arrayed echoendoscope. Dig Endosc 2011;23 Suppl 1:2-8.

9. Hara K, Bhatia V, Hijioka S, Mizuno N, Yamao K. A convex EUS is useful to diagnose vascular invasion of cancer, especially hepatic hilus cancer. Dig Endosc 2011;23 Suppl 1:26-28.

10. Kaneko M, Katanuma A, Maguchi H, et al. Prospective, randomized, comparative study of delineation capability of radial scanning and curved linear array endoscopic ultrasound for the pancreaticobiliary region. Endosc Int Open 2014;2:E160-E170.

11. Ito K, Fujita N, Noda Y, et al. Preoperative evaluation of ampullary neoplasm with EUS and transpapillary intraductal US: a prospective and histopathologically controlled study. Gastrointest Endosc 2007;66:740747. 